\title{
Disentangling different structures in heavy-light four-quark states
}

\author{
Paul C. Wallbott, ${ }^{1, *}$ Gernot Eichmann $\oplus^{2,3, \dagger}$ and Christian S. Fischer $\oplus^{1,4, *}$ \\ ${ }^{1}$ Institut für Theoretische Physik, Justus-Liebig Universität Gießen, 35392 Gießen, Germany \\ ${ }^{2}$ LIP Lisboa, Av. Prof. Gama Pinto 2, 1649-003 Lisboa, Portugal \\ ${ }^{3}$ Departamento de Física, Instituto Superior Técnico, 1049-001 Lisboa, Portugal \\ ${ }^{4}$ Helmholtz Research Academy Hesse for FAIR (HFHF), Campus Gießen, 35392 Gießen, Germany
}

(Received 23 June 2020; accepted 24 August 2020; published 25 September 2020)

\begin{abstract}
Models proposed to explain recently discovered heavy-light four-quark states already assume certain internal structures, i.e., the (anti)quark constituents are grouped into diquark/antidiquark clusters, heavymeson/light-meson clusters (hadrocharmonium) or heavy-light meson molecules. We propose and use an approach to four-quark states based on Dyson-Schwinger and Bethe-Salpeter equations that has the potential to discriminate between these models. We study the masses of heavy-light $c q \bar{q} \bar{c}$ and $c c \bar{q} \bar{q}$ four-quark states with $q=u, d, s$ and quantum numbers $I\left(J^{P C}\right)=0\left(1^{++}\right), 1\left(1^{+-}\right), 0\left(0^{++}\right)$and $1\left(0^{+}\right), 0\left(1^{+}\right), 1\left(1^{+}\right)$. We identify the dominant components of the ground states with these quantum numbers and suggest candidates for corresponding experimental states. Most notably, we find strong heavy-light meson-meson and negligible diquark-antidiquark components in all $c q \bar{q} \bar{c}$ states, whereas for $c c \bar{q} \bar{q}$ states diquarks are present. A potential caveat in the $I=0$ channels is the necessary but costly inclusion of $c \bar{c}$ components which is relegated to future work.
\end{abstract}

DOI: 10.1103/PhysRevD.102.051501

\section{INTRODUCTION}

In the past two decades a number of highly interesting states have been identified in the charmonium and bottomonium energy regions that cannot be accommodated for in the conventional quark model for mesons made of a quark and an antiquark. Since the quark model is otherwise extremely successful in predicting spectra of heavy $Q \bar{Q}$ states (with $Q=c, b$ ), these exceptional states are considered to be exotic hadrons. Some of them carry electromagnetic charge and thus may be naturally explained as four-quark states $Q \bar{Q} q \bar{q}(q=u, d, s)$ with a light charged quark-antiquark pair in addition to the overall neutral $Q \bar{Q}$ component. Thus, four-quark states are considered as promising candidates to explain the properties of these exotic hadrons, see e.g., [1-7] for reviews.

There is, however, no agreement on the internal structure of these four-quark states. Model approaches usually assume some kind of internal clustering from the start. One possibility, the hadroquarkonium picture [8], suggests a heavy quark and antiquark grouped together in a tight

\footnotetext{
*paul.wallbott@physik.uni-giessen.de gernot.eichmann@tecnico.ulisboa.pt christian.fischer@physik.uni-giessen.de
}

Published by the American Physical Society under the terms of the Creative Commons Attribution 4.0 International license. Further distribution of this work must maintain attribution to the author(s) and the published article's title, journal citation, and DOI. Funded by SCOAP. core surrounded by the light $q \bar{q}$ pair. This is motivated by the experimental observation of final states with a specific charmonium state and light hadrons. The second possibility is the clustering of constituents in diquark-antidiquark $(d q-\overline{d q})$ components which interact via colored forces, see e.g., [1] for a review. A third possibility, especially relevant for states close to open-charm thresholds, is the meson-molecule picture of arrangements into pairs of $D^{(*)} \bar{D}^{(*)}$ mesons that interact with each other by shortand/or long-range forces [5].

It is important to note that these possibilities are not mutually exclusive: In general, every experimental state may be a superposition of components with a different structure and the "leading" component may be different on a case-by-case basis. It is therefore important to develop theoretical approaches to QCD that can deal with all these possibilities. Lattice QCD is one such approach and has made interesting progress so far, see [9-18] and references therein. Nevertheless most simulations have been performed at an exploratory level using light quarks with unphysical large masses. Functional methods, on the other hand, have been restricted to four-quark states with equal masses [19,20] or specific quantum numbers [21].

In this work we present a generalization of the functional approach to four-quark states that has the potential to systematically address and compare heavy-light states in different flavor combinations and with different $J^{P C}$ quantum numbers. Based on a well-studied and understood truncation of the underlying quark-gluon interaction, we work with an approximated version of the four-body 
Faddeev-Yakubovsky equations that takes into account the two-body correlations that lead to the internal clustering described above. We apply the resulting formalism to the experimentally interesting $c q \bar{q} \bar{c}$ hidden-charm states with quantum numbers $J^{P C}=0\left(1^{++}\right), 1\left(1^{+-}\right)$and $0\left(0^{++}\right)$, which are carried by the $X(3872)$ [22,23], the neutral $Z(3900)$ [24] and (likely) the $X(3915)$ [25], respectively. Furthermore, we discuss four-quark states with open charm $c c \bar{q} \bar{q}$ in the channels $1\left(0^{+}\right), 0\left(1^{+}\right)$and $1\left(1^{+}\right)$. Currently there are no experimental candidates for these states but searches are underway. Corresponding states in the heavier bottom-quark region received a lot of attention in recent years since they are promising candidates for deeply bound and narrow states, see e.g., $[15,16,26,27]$. It is certainly interesting to see whether this is still the case for the experimentally more easily accessible open-charm states.

\section{FOUR-BODY EQUATION}

The homogeneous Bethe-Salpeter equation (BSE) shown in Fig. 1 has the form

$$
\Gamma=K G_{0} \Gamma
$$

where $\Gamma$ is the BS amplitude, $K$ is the four-quark interaction kernel that contains all possible two-, three- and four-body interactions, and $G_{0}$ is the product of four dressed (anti) quark propagators; see [19-21] for details. Each multiplication represents an integration over all loop momenta. Equation (1) holds at a given pole position of the offshell $q q \overline{q q}$ scattering matrix $T$, which satisfies the scattering equation $T=K+K G_{0} T$. Poles on the real axis of the total squared momentum $P^{2}$ correspond to bound states, whereas resonances appear as poles in the complex plane on higher Riemann sheets.

In this work we focus entirely on the two-body correlations in $K$ since these generate the internal two-body clusters discussed above. This leads to

$$
K G_{0}=\sum_{a a^{\prime}} K_{a a^{\prime}}, \quad K_{a a^{\prime}}=K_{a}+K_{a^{\prime}}-K_{a} K_{a^{\prime}}
$$

where $a, a^{\prime}$ stand for $q q, \bar{q} \bar{q}$ or $q \bar{q}$ pairs and $a a^{\prime}$ is either (12)(34), (13)(24) or (14)(23). The subtraction is necessary to avoid overcounting $[19,28,29]$. Irreducible three- and four-body interactions are not (yet) taken into account for three reasons: first, this would complicate an already tremendous numerical task further beyond the resources

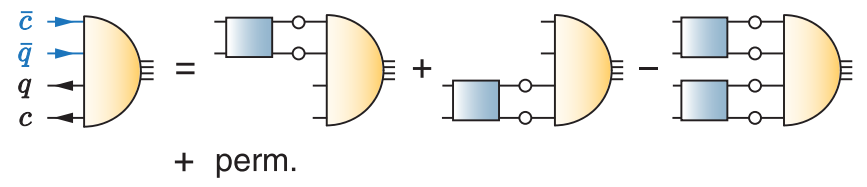

FIG. 1. Four-quark BSE for a $c q \bar{q} \bar{c}$ system in the (12)(34) configuration; the remaining (13)(24) and (14)(23) permutations are not shown. The half-circles and boxes represent the tetraquark amplitude and Bethe-Salpeter kernel, respectively. currently available to us; second, a similar strategy has been employed with great success in the baryon sector, where strong two-body correlations naturally lead to a diquarkquark picture, which in turn leads to a spectrum in one-toone agreement with experiment [30,31]; third and most important, the pictures of internal structures that we like to discriminate (i.e., diquark/antidiquark vs hadro-charmonium vs meson molecule) all rely on strong two-body clustering. Thus for the purpose of this work it is indeed sufficient to focus on two-body interactions. Nevertheless, of course, the effects of irreducible three- and four-body forces have to be explored in future work.

For the two-body kernels we employ the same rainbowladder interaction that is used in the Dyson-Schwinger equation (DSE) for the quark propagator. This truncation has recently been reviewed in [31], where the DSE for the quark propagator is discussed around Eq. (3.18) and the effective interaction in Eqs. (3.95)-(3.96). We use $\Lambda=$ $0.72 \mathrm{GeV}$ for the scale parameter, adjusted to reproduce the pion decay constant $f_{\pi}$, and $\eta=1.8 \pm 0.2$. Together with the current-quark masses, these are the only input parameters in all equations. The construction satisfies chiral constraints such as the Gell-Mann-Oakes-Renner relation, ensures the (pseudo-) Goldstone-boson nature of the pion and has been extensively applied to meson and baryon phenomenology. As discussed in [31], the truncation is well known to reliably reproduce many properties of pseudoscalar and vector mesons (and, correspondingly, scalar and axialvector diquarks). Since we focus on two-body clusters inside tetraquarks in these channels only, we may expect qualitatively reasonable results.

The quantitative reliability of the approximation of the two-body kernel may be judged from the results for meson masses in Table I. We work in the isospin symmetric limit where $m_{D^{+}}=m_{D^{-}}=m_{D^{0}}$. The $u / d$ current-quark mass is fixed by $m_{\pi}$, the strange quark mass is chosen such that the sum $m_{D_{s}}+m_{D_{s}^{*}}$ equals the sum of the experimental values [32] and analogously for the charm quark mass in $m_{D}+m_{D^{*}}$. The deviations between the theoretical and experimental meson masses are then below $7 \%$ in all cases.

TABLE I. Rainbow-ladder results for $n \bar{n}, c \bar{n}, c \bar{s}$ and $c \bar{c}$ meson and diquark masses (in $\mathrm{MeV} ; n=u, d$ ). $m_{\bar{q}}$ is the input currentquark mass at a renormalization point $\mu=19 \mathrm{GeV}$ in a momentum-subtraction scheme. The column $m_{P S}$ contains the masses of $\pi, D, D_{s}$ and $\eta_{c}$, the column $m_{V}$ those of $\rho / \omega, D^{*}$, $D_{s}^{*}$ and $J / \psi$, and the columns $m_{S}$ and $m_{A}$ list the corresponding diquark masses. The quoted errors are obtained by varying the parameter $\eta=1.8 \pm 0.2$.

\begin{tabular}{ccccrc}
\hline \hline & $m_{\bar{q}}$ & $m_{P S}$ & $m_{V}$ & \multicolumn{1}{c}{$m_{S}$} & $m_{A}$ \\
\hline$n \bar{n}$ & 3.7 & 138 & $732(1)$ & $802(77)$ & $999(60)$ \\
$c \bar{n}$ & 3.7 & $1802(2)$ & $2068(16)$ & $2532(90)$ & $2572(8)$ \\
$c \bar{s}$ & 91 & $1911(3)$ & $2169(14)$ & $2627(82)$ & $2666(7)$ \\
$c \bar{c}$ & 795 & $2792(6)$ & $2980(6)$ & $3382(15)$ & $3423(8)$ \\
\hline \hline
\end{tabular}




\section{FOUR-QUARK AMPLITUDE}

The main challenge in solving Eq. (1) for given $J^{P C}$ is the structure of the BS amplitude. Its general decomposition can be written as

$$
\Gamma_{\alpha \beta \gamma \delta}^{(\mu)}\left(p_{1} \ldots p_{4}\right)=\sum_{i} f_{i}(\ldots) \tau_{i}^{(\mu)}\left(p_{1} \ldots p_{4}\right)_{\alpha \beta \gamma \delta},
$$

where the Lorentz-invariant dressing functions $f_{i}(\ldots)$ depend on the ten Lorentz invariant momentum variables that can be constructed from four independent momenta. The tensors $\tau_{i}$ are the direct products of Dirac, color and flavor parts. A $J=0$ state has 256 linearly independent Dirac tensors and a $J=1$ state 768 , which are collected in Ref. [20] and the Appendix of [21]. The color part of the amplitude consists of two independent color-singlet tensors and the flavor wave functions depend on the particular system, cf. [33] for details.

To extract physical content from the BS amplitude $\Gamma^{(\mu)}\left(p_{1} \ldots p_{n}\right)$, we observe that the amplitude develops internal two-body clusters, which for heavy-light systems occur in the three different channels corresponding to hadroquarkonia, heavy-light meson-meson components and $d q-\overline{d q}$ clusters. These clusters may go on-shell provided that the sum of their masses is smaller than the mass of the four-body state. If this occurs in color-singlet channels, the four-quark state becomes a resonance in the two-body hadronic system of the corresponding clusters. But even if the masses of the two-body clusters are large enough such that the probed momenta only come close to the corresponding singularities, this will influence the four-body system. Thus the guiding idea is to represent $\Gamma^{(\mu)}\left(p_{1} \ldots p_{n}\right)$ in terms of these two-body clusters.

Since we are interested in specific quantum numbers with experimental candidates for four-quark states, we draw on existing information on the decay channels of these states and construct our representation along the content displayed in Table II. For example, in the heavylight meson sector we took into account combinations of the $I\left(J^{P}\right)=1 / 2\left(0^{-}\right)$multiplets $\left(D, D^{*}\right)$ and their antiparticles $\left(\bar{D}, \bar{D}^{*}\right)$, omitting the heavy combination $D^{*} \bar{D}^{*}$. Note that since we work in the isospin-symmetric limit the charged and neutral states are mass-degenerate and both taken into account.

TABLE II. Physical content of the BS amplitudes for flavor combinations $c q \overline{q c}$ and $c c \overline{q q}$. Scalar and axialvector diquarks are denoted by $S$ and $A$, respectively.

\begin{tabular}{ccc}
\hline \hline & $I\left(J^{P(C)}\right)$ & Physical components \\
\hline$c q \overline{q c}$ & $0\left(0^{++}\right)$ & $D D, J / \psi \omega, S S$ \\
& $0\left(1^{++}\right)$ & $D D^{*}, J / \psi \omega, S A$ \\
& $1\left(1^{+-}\right)$ & $D D^{*}, J / \psi \pi, S A$ \\
& $1\left(0^{+}\right)$ & $D D, A A$ \\
& $0\left(1^{+}\right)$ & $D D^{*}, A S$ \\
& $1\left(1^{+}\right)$ & $D D^{*}, A A$ \\
\hline \hline
\end{tabular}

The construction of the $\tau_{i}^{(\mu)}\left(p_{1} \ldots p_{4}\right)$ for the configurations $q q \overline{q q}, c q \overline{q c}$ and $c c \overline{q q}$ is detailed in [33]. We construct the Dirac parts according to the dominant two-body clusters and combine them with appropriate color and flavor wave functions such that the charge-conjugation and Pauli exchange symmetries are respected. As a result, one populates a physically motivated subset of all possible basis elements. Whereas in this work we restrict ourselves to the combinations displayed in Table II, in principle one could construct a complete basis for the four-body amplitude with entangled Dirac, color and flavor tensors including all possible meson and diquark channels (i.e., also those with higher total angular momentum).
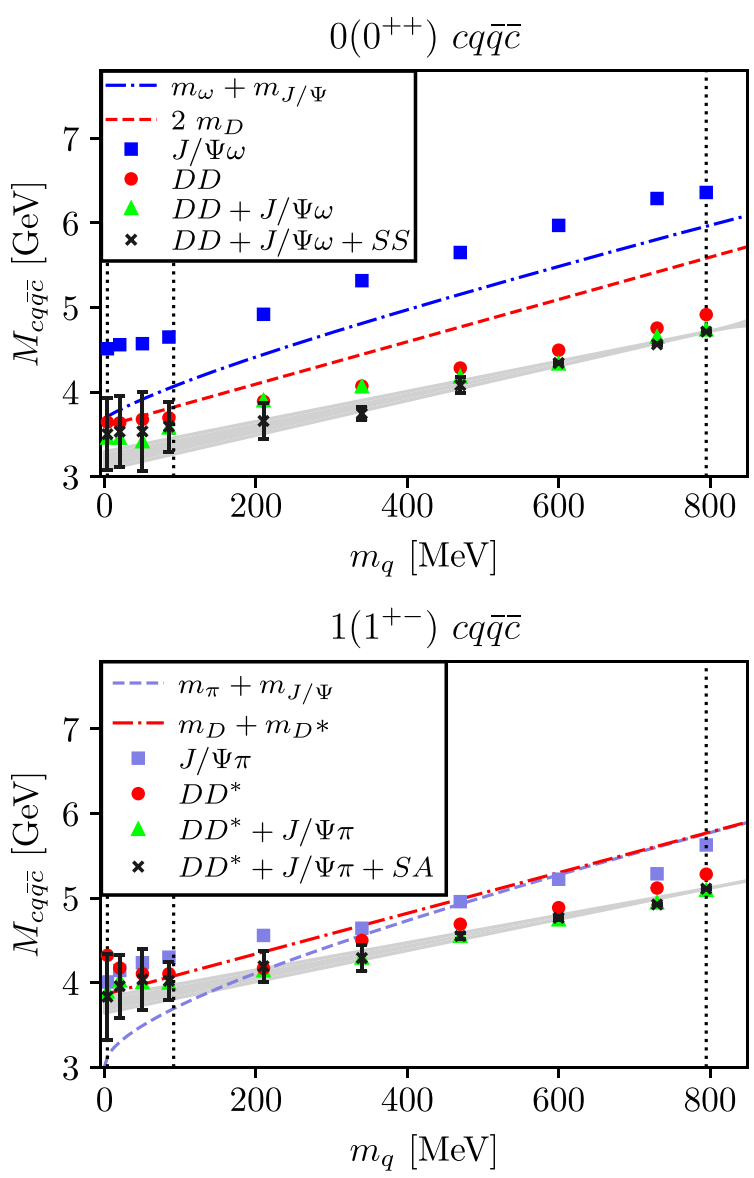

FIG. 2. Quark-mass evolution of the $c q \overline{q c}$ ground states in the $0\left(0^{++}\right)$and $1\left(1^{+-}\right)$channels for different components of the fourbody amplitude. The three vertical dashed lines mark the positions of the up/down, strange and charm quark (from left to right). Masses below the respective two-meson thresholds have been determined directly from the eigenvalue curve $\lambda\left(P^{2}\right)$ of the BSE. Results above the threshold (i.e., for small quark masses) are obtained from extrapolated eigenvalue curves. For example, in the upper plot, the masses obtained with $J / \psi \omega$ components only are all extrapolated, whereas in the other three setups all results are read off directly, except those at the smallest quark masses which lie above the lowest threshold. See main text for further details. 
With this setup, we are in a position to solve the BSEs as an eigenvalue problem, with structure $\lambda \Gamma=K G_{0} \Gamma$ and general eigenvalue $\lambda$. All elements of this equation depend explicitly on the total momentum $P$. By varying $P^{2}$ such that $\lambda=1$ one finds the mass of the bound state/resonance in the four-body system via $P^{2}=-M^{2}$. One problem that appears in this search is the potential appearance of singularities in the plane of the complex total momentum due to the internal meson and diquark correlations. Typically, this does not happen for large masses of the lighter quark pair, where the resulting bound state is in general (well) below the meson-meson thresholds, cf. Figs. 2 and 3. However, this situation changes for smaller masses and in particular close to the physical point of light quark masses, where the mass of the bound state/ resonance comes close to or is even larger than the mesonmeson threshold. For these cases we determine the eigenvalue curve $\lambda\left(M^{2}\right)$ in the singularity free region and
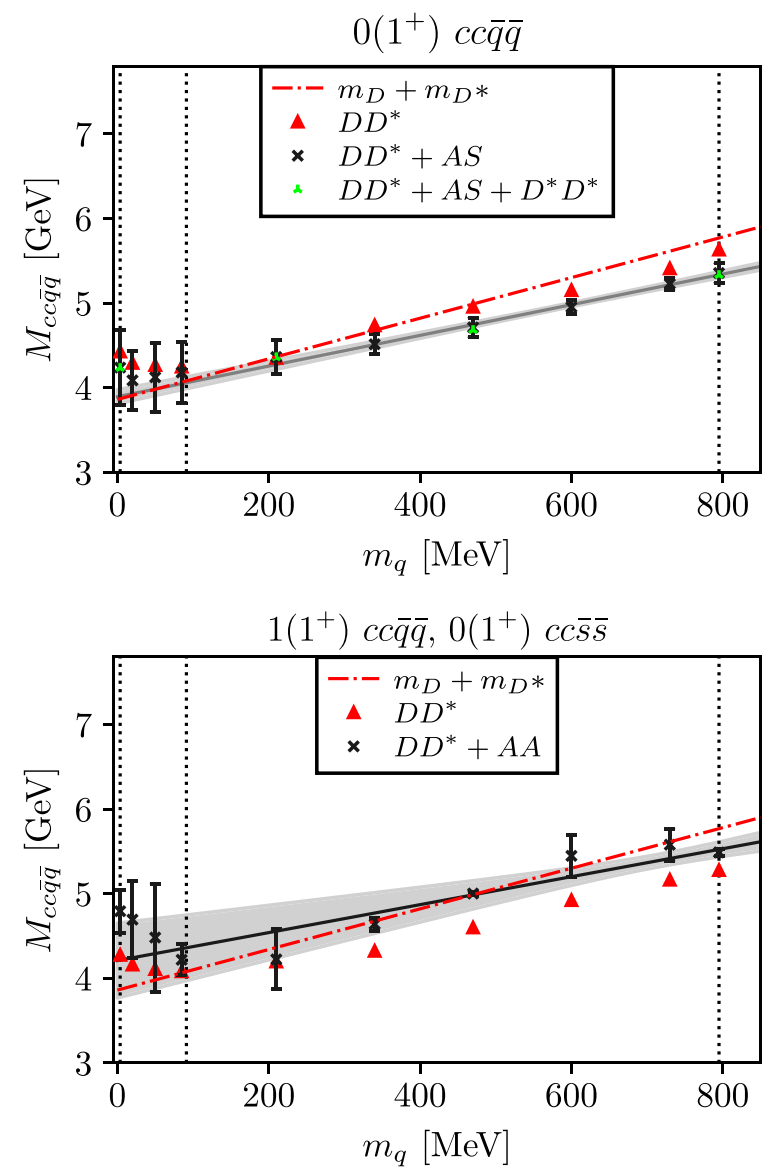

FIG. 3. Quark-mass evolution of the $c c \overline{q q}$ ground states in the $0\left(1^{+}\right)$and $1\left(1^{+}\right)$channels. The $0\left(1^{+}\right) c c \overline{s s}$ state is read off from the curve for the $1\left(1^{+}\right)$state at $m_{q}=m_{s}$. The three vertical dashed lines mark the positions of the up/down, strange and charm quark (from left to right). Masses below the $D D^{*}$ threshold have been determined directly from the eigenvalue curve $\lambda\left(P^{2}\right)$ of the BSE. Results above the threshold (i.e., for small quark masses) are obtained from extrapolated eigenvalue curves. extrapolate it further into the timelike momentum domain using rational functions. This procedure can only pick up the real part of potentially complex masses, i.e., it is not possible to extract decay widths. To this end one would need to use the much more involved approach described in [35,36], which is not (yet) at our disposal for heavy-light four-quark states. For many further details and first results in the light quark sector we refer the reader to Ref. [34].

\section{HIDDEN-CHARM STATES}

We first discuss our results for the hidden-charm $c q \overline{q c}$ four-quark states in the $I\left(J^{P C}\right)=0\left(0^{++}\right)$and $1\left(1^{+-}\right)$ channels. In this context we wish to emphasise that the results in the $I=0$-channel must be seen as preliminary since the necessary but costly inclusion of $c \bar{c}$ components in our approach is relegated to future work (see however [34] for corresponding results in the light quark sector).

The results for the $0\left(1^{++}\right)$heavy-light state can be found in [21], where we also described our procedure to estimate (part of) the error of the calculation. In that case we found a dominant heavy-light meson component, whereas the hadrocharmonium component is rather weak and the diquark component has almost no effect at all.

A similar pattern arises in the scalar $0\left(0^{++}\right)$case displayed in the top panel of Fig. 2. We show the mass evolution of the four-quark state when the mass of the $c \bar{c}$ pair is fixed and the mass of the other $q \bar{q}$ pair is varied from the charm mass (rightmost vertical dashed line) to the strange and light quark masses (other two vertical lines). We compare calculations with hadrocharmonium content only ( $J / \psi \omega$; squares), heavy-light meson components only ( $D D$; circles) and a combination of the two (triangles). The full calculation including also the diquark/antidiquark component $(D D+J / \psi \omega+S S)$ is marked with crosses. Not contained in the figure is our result for using diquark components only $(S S)$. For the scalar channel and in fact for all channels that we studied we obtain very large masses with diquarks only. These are typically of the order of the diquark/antidiquark thresholds around $5 \mathrm{GeV}$ (cf. Table I) and therefore unphysical. Comparing the results for $0\left(0^{++}\right)$ shown in Fig. 2 we find that for large masses, the $d q-\overline{d q}$ component has almost no effect on the results, whereas the hadrocharmonium component gives only mild corrections to the leading heavy-light meson components. The diquark corrections become somewhat more prominent for small masses, however without changing the general picture. Due to the sizeable error bar we cannot discriminate between a bound state and a resonance. The masses quoted in Table III are obtained from a linear fit to the mass evolution at larger quark masses; only for $0\left(0^{++}\right)$the direct calculation yields a mass below any threshold.

The general picture changes somewhat for the $1\left(1^{+-}\right)$ state shown at the bottom of Fig. 2. Once again we find negligible diquark components and a strong heavy-light meson component, but also non-negligible contributions 
from the hadrocharmonium component. It is interesting to compare this behavior to the $1^{++}$channel discussed in [21]. From Table II we observe that the only difference between the two states are the hadrocharmonium components. The $J / \psi \pi$ component in the $1^{+-}$channel is significantly lighter than the $J / \psi \omega$ component in the $1^{++}$channel, which lifts the degeneracy between the two states and leads to a lighter mass of the $1^{+-}$(which is, however, opposite to the current experimental situation). We have also tested further components, which can contribute to the axial-vector $1\left(1^{+-}\right)$ state. We found the $D^{*} D^{*}$ components to be negligible; however, the $\eta_{c} \rho$ component is sizeable and enhances the mass splitting once included. Further studies in this direction are necessary.

The resulting masses are collected in Table III. It is interesting to compare our results with expectations from the literature. In Ref. [37] heavy-quark symmetry has been used to predict patterns for molecular states. This led to the identification of the $X(3872)$ with a molecular state in the $1^{++}$channel and the neutral $Z_{c}(3900)$ with a molecular state in the $1^{+-}$channel. Our results agree with this identification: for the $X(3872)$ we expect an almost pure heavy-light meson state which is then natural to expect to sit very close to the $D \bar{D}^{*}$ threshold. For the $Z_{c}(3900)$, however, we find non-negligible corrections from other components, which may shift the physical state away from the threshold. For the scalar channel no predictions have been made in [37], see however [38-40] for a detailed discussion. The lightest scalar molecule, if it exists, would be expected at the $D \bar{D}$ threshold. This is indeed the case for our scalar state, which sits in the region of the threshold of our $D$ mesons (cf. Table I). Thus the mass pattern emerging in Table III is in line with our observation of heavy-light meson dominance in all $c q \bar{q} \bar{c}$ states studied so far.

In Table III we also list the masses of the charm-strange $\operatorname{cs} \bar{s} \bar{c}$ states extracted from the mass evolution. For $I=0$ these correspond to observable states, whereas for $I=1$ they are unphysical. In the $1^{++}$channel there is an experimental candidate, namely the $X(4140)$ with a mass only slightly above the upper range of our error bar. Provided this identification holds, we predict a strong

TABLE III. Masses of hidden-charm $(c q \overline{q c})$ and open-charm $(c c \overline{q q})$ states $(n=u, d)$ in $\mathrm{GeV}$. The combined error from fitting and varying the momentum partitioning is given in parentheses. For the $0\left(0^{++}\right)$state we quote both the fitted value (first line) and the direct calculation (second line). Tetraquarks with open or hidden strangeness are only quoted in channels where physical states may be present.

\begin{tabular}{lccccc}
\hline \hline$I\left(J^{P C}\right)$ & $c n \overline{n c}$ & $c s \overline{s c}$ & $I\left(J^{P}\right)$ & $c c \overline{n n}$ & $c c \overline{s s}$ \\
\hline $0\left(0^{++}\right)$ & $3.20(11)$ & $3.36(10)$ & $0\left(0^{+}\right)$ & $\ldots$ & $3.95(10)$ \\
& $3.50(42)$ & $3.59(30)$ & $1\left(0^{+}\right)$ & $3.80(10)$ & $\ldots$ \\
$0\left(1^{++}\right)$ & $3.92(7)$ & $4.07(6)$ & $0\left(1^{+}\right)$ & $3.90(8)$ & $4.36(39)$ \\
$1\left(1^{+-}\right)$ & $3.74(9)$ & $\ldots$ & $1\left(1^{+}\right)$ & $4.22(44)$ & $\ldots$ \\
\hline \hline
\end{tabular}

heavy-light meson component of the $X(4140)$, even though it is not overly close to the $D_{s} \bar{D}_{s}^{*}$ threshold. In addition, we find a corresponding state in the $0^{++}$channel, although the large error bars in this case make a prediction of its mass rather imprecise.

\section{OPEN-CHARM STATES}

Our results for the open-charm states with flavor content $c c \overline{q q}$ are shown in Fig. 3. The basis construction in the open-charm case is significantly different from hidden charm since charge-conjugation symmetry is replaced by Pauli antisymmetry. The two heavy-light meson combinations $(c \bar{q})(c \bar{q})$ are identical, whereas the $d q-\overline{d q}$ component $(c c)(\bar{q} \bar{q})$ with a heavy diquark and a light antidiquark inherits the role of the hadrocharmonium component. For $I=0$, the light $\bar{q} q$ antidiquark must be scalar $(S)$ due to symmetry, whereas for $I=1$ it is axial-vector $(A)$. The heavy $c c$ pair is always an axial-vector diquark.

This change of internal dynamics is also reflected in the results. The heavy-light meson component alone produces a state that is well below the $D D^{*}$ threshold and moves only slightly above threshold for decreasing quark masses. Whereas the $A S$ diquark contribution in the $I=0$ case is negligible compared to the $D D^{*}$ contribution, the $A A$ diquark component for $I=1$ has a significant impact in pushing the mass evolution up above threshold. In the graph for $0\left(1^{+}\right)$we also included the $D^{*} \bar{D}^{*}$ component explicitly, which is negligible as in all other channels. The resulting mass hierarchy between the isosinglet and isotriplet states is as expected from heavy quark symmetry [26]. Our extrapolated values for the masses are in the ballpark expected from other approaches, see e.g., $[26,27,41]$ and references therein.

In the open-charm case, the $c c \overline{s s}$ states (with $I=0$ ) must be read off from the $c c \overline{q q}$ curves with $I=1$ since those have the same wave-function components [33]. As a consequence, several slots in Table III are empty because they do not support physical states. Moreover, there is a large gap between the light and strange state in the $0\left(1^{+}\right)$ channel but it comes again with a sizeable mass uncertainty.

\section{CONCLUSIONS}

In this work we have studied and compared the masses of heavy-light four-quark states in the charm energy region. We developed a dynamical framework that takes into account all possible combinations of internal two-body clusters and is therefore able to decide dynamically whether pictures from effective field theory and models (meson molecule, hadrocharmonium, diquark-antidiquark) are realized. For hidden charm, in all cases considered we do not find a sizeable diquark-antidiquark component. Instead, the heavy-light meson component is favoured, with channel-dependent negligible [for $\left.0\left(1^{++}\right)\right]$or small but significant $\left[0\left(0^{++}\right)\right.$ and $1\left(1^{+-}\right)$] contributions from the hadrocharmonium 
component. The situation for open charm is similar; the dominant contribution is the heavy-light meson component although significant corrections from the diquark-antidiquark component arise. Although the masses of the charm quarks are far from static, mass patterns expected from heavy-quark symmetry are visible: In the open-charm sector we observe the expected mass hierarchy and difference between the $I=0$ and $I=1$ axial-vector channels [26]. The observed mass pattern in the hidden charm sector resembles the one expected of the lowest-lying multiplet of states in the hadronic molecular approach [37]. In order to make further contact with heavy-quark symmetry and lattice QCD, it would be interesting to further increase the masses of the heavy quarks and explore the bottomonium sector. This is technically challenging and therefore left for future studies. A potential caveat of the present formalism is that it does not take into account potentially important effects from mixing with ordinary $c \bar{c}$ states in the $I=0$ channels $[10,14]$. Again, this is left for future work.

\section{ACKNOWLEDGMENTS}

We are grateful to Christoph Hanhart, Soeren Lange, Sasa Prelovsek and Marc Wagner for discussions. This work was supported by the DFG Grant No. FI 970/11-1 and by the FCT Investigator Grant No. IF/00898/2015.
[1] A. Esposito, A. Pilloni, and A. D. Polosa, Phys. Rep. 668, 1 (2017).

[2] R. F. Lebed, R. E. Mitchell, and E. S. Swanson, Prog. Part. Nucl. Phys. 93, 143 (2017).

[3] H.-X. Chen, W. Chen, X. Liu, and S.-L. Zhu, Phys. Rep. 639, 1 (2016).

[4] A. Ali, J. S. Lange, and S. Stone, Prog. Part. Nucl. Phys. 97, 123 (2017).

[5] F.-K. Guo, C. Hanhart, U.-G. Meißner, Q. Wang, Q. Zhao, and B.-S. Zou, Rev. Mod. Phys. 90, 015004 (2018).

[6] S. L. Olsen, T. Skwarnicki, and D. Zieminska, Rev. Mod. Phys. 90, 015003 (2018).

[7] Y.-R. Liu, H.-X. Chen, W. Chen, X. Liu, and S.-L. Zhu, Prog. Part. Nucl. Phys. 107, 237 (2019).

[8] M. B. Voloshin, Prog. Part. Nucl. Phys. 61, 455 (2008).

[9] S. Prelovsek, T. Draper, C. B. Lang, M. Limmer, K.-F. Liu, N. Mathur, and D. Mohler, Phys. Rev. D 82, 094507 (2010).

[10] S. Prelovsek and L. Leskovec, Phys. Rev. Lett. 111, 192001 (2013).

[11] J. Berlin, A. Abdel-Rehim, C. Alexandrou, M. Dalla Brida, M. Gravina, and M. Wagner, Proc. Sci., LATTICE2014 (2014) 104 [arXiv:1410.8757].

[12] S.-h. Lee, C. DeTar, H. Na, and D. Mohler (Fermilab Lattice, MILC Collaborations), arXiv:1411.1389.

[13] S. Prelovsek, C. B. Lang, L. Leskovec, and D. Mohler, Phys. Rev. D 91, 014504 (2015).

[14] M. Padmanath, C. B. Lang, and S. Prelovsek, Phys. Rev. D 92, 034501 (2015).

[15] A. Francis, R. J. Hudspith, R. Lewis, and K. Maltman, Phys. Rev. Lett. 118, 142001 (2017).

[16] P. Bicudo, M. Cardoso, A. Peters, M. Pflaumer, and M. Wagner, Phys. Rev. D 96, 054510 (2017).

[17] A. Francis, R. J. Hudspith, R. Lewis, and K. Maltman, Phys. Rev. D 99, 054505 (2019).

[18] L. Leskovec, S. Meinel, M. Pflaumer, and M. Wagner, Phys. Rev. D 100, 014503 (2019).

[19] W. Heupel, G. Eichmann, and C. S. Fischer, Phys. Lett. B 718, 545 (2012).

[20] G. Eichmann, C. S. Fischer, and W. Heupel, Phys. Lett. B 753, 282 (2016).
[21] P. C. Wallbott, G. Eichmann, and C. S. Fischer, Phys. Rev. D 100, 014033 (2019).

[22] S. K. Choi et al. (Belle Collaboration), Phys. Rev. Lett. 91, 262001 (2003).

[23] R. Aaij et al. (LHCb Collaboration), Phys. Rev. Lett. 110, 222001 (2013).

[24] M. Ablikim et al. (BESIII Collaboration), Phys. Rev. Lett. 115, 112003 (2015).

[25] K. Abe et al. (Belle Collaboration), Phys. Rev. Lett. 94, 182002 (2005).

[26] E. J. Eichten and C. Quigg, Phys. Rev. Lett. 119, 202002 (2017).

[27] M. Karliner and J. L. Rosner, Phys. Rev. Lett. 119, 202001 (2017).

[28] K. Huang and H. A. Weldon, Phys. Rev. D 11, 257 (1975).

[29] A. M. Khvedelidze and A. N. Kvinikhidze, Theor. Math. Phys. 90, 62 (1992).

[30] G. Eichmann, C. S. Fischer, and H. Sanchis-Alepuz, Phys. Rev. D 94, 094033 (2016).

[31] G. Eichmann, H. Sanchis-Alepuz, R. Williams, R. Alkofer, and C. S. Fischer, Prog. Part. Nucl. Phys. 91, 1 (2016).

[32] M. Tanabashi et al. (Particle Data Group Collaboration), Phys. Rev. D 98, 030001 (2018).

[33] See Supplemental Material at http://link.aps.org/ supplemental/10.1103/PhysRevD.102.051501 for the construction of the amplitudes.

[34] N. Santowsky, G. Eichmann, C. S. Fischer, P. C. Wallbott, and R. Williams, arXiv:2007.06495.

[35] R. Williams, Phys. Lett. B 798, 134943 (2019).

[36] G. Eichmann, P. Duarte, M. Pea, and A. Stadler, Phys. Rev. D 100, 094001 (2019).

[37] M. Cleven, F.-K. Guo, C. Hanhart, Q. Wang, and Q. Zhao, Phys. Rev. D 92, 014005 (2015).

[38] D. Gamermann, E. Oset, D. Strottman, and M. V. Vacas, Phys. Rev. D 76, 074016 (2007).

[39] D. Gamermann and E. Oset, Eur. Phys. J. A 36, 189 (2008).

[40] L. Dai, J.-J. Xie, and E. Oset, Eur. Phys. J. C 76, 121 (2016).

[41] S.-Q. Luo, K. Chen, X. Liu, Y.-R. Liu, and S.-L. Zhu, Eur. Phys. J. C 77, 709 (2017). 